\title{
Oscillation Criteria for Certain Even Order Neutral Delay Differential Equations with Mixed Nonlinearities
}

\author{
Zhen-Lai Han, Yi-Bing Sun, Yan Zhao, and Dian-Wu Yang \\ School of Mathematical Sciences, University of Jinan, Jinan, Shandong 250022, China \\ Correspondence should be addressed to Zhen-Lai Han; hanzhenlai@163.com
}

Received 16 March 2014; Accepted 21 June 2014; Published 3 August 2014

Academic Editor: Tongxing Li

Copyright (C) 2014 Zhen-Lai Han et al. This is an open access article distributed under the Creative Commons Attribution License, which permits unrestricted use, distribution, and reproduction in any medium, provided the original work is properly cited.

We establish some oscillation criteria for the following certain even order neutral delay differential equations with mixed nonlinearities: $\left(r(t)\left|z^{(n-1)}(t)\right|^{\alpha-1} z^{(n-1)}(t)\right)+q_{0}(t) \mid\left(\left.x\left(\tau_{0}(t)\right)\right|^{\alpha-1} x\left(\tau_{0}(t)\right)+q_{1}(t) \mid\left(\left.x\left(\tau_{1}(t)\right)\right|^{\beta-1} x\left(\tau_{1}(t)\right)+q_{2}(t) \mid\left(\left.x\left(\tau_{2}(t)\right)\right|^{\gamma-1} x\left(\tau_{2}(t)\right)=\right.\right.\right.$ $0, t \geq t_{0}$, where $z(t)=x(t)+p(t) x(\sigma(t)), n$ is even integer, and $\gamma>\alpha>\beta>0$. Our results generalize and improve some known results for oscillation of certain even order neutral delay differential equations with mixed nonlinearities.

\section{Introduction}

In this paper, we are concerned with oscillation behavior of the certain even order neutral delay differential equations with mixed nonlinearities:

$$
\begin{aligned}
&\left(r(t)\left|z^{(n-1)}(t)\right|^{\alpha-1} z^{(n-1)}(t)\right)^{\prime} \\
&+q_{0}(t)\left|x\left(\tau_{0}(t)\right)\right|^{\alpha-1} x\left(\tau_{0}(t)\right) \\
&+q_{1}(t)\left|x\left(\tau_{1}(t)\right)\right|^{\beta-1} x\left(\tau_{1}(t)\right) \\
&+q_{2}(t)\left|x\left(\tau_{2}(t)\right)\right|^{\gamma-1} x\left(\tau_{2}(t)\right)=0, \\
& t \geq t_{0},
\end{aligned}
$$

where $z(t)=x(t)+p(t) x(\sigma(t)), n$ is even integer, and $\gamma>$ $\alpha>\beta>0$ are constants. $r, q_{i} \in C\left(\left[t_{0}, \infty\right), R^{+}\right), r^{\prime}(t) \geq 0$, $p, \tau_{i} \in C\left(\left[t_{0}, \infty\right), R\right)$ satisfy that $\tau_{i}(t) \leq t, i=0,1,2$, and there exists a function $\sigma \in C\left(\left[t_{0}, \infty\right), R\right)$, such that $\sigma(t) \leq t$, $\lim _{t \rightarrow \infty} \sigma(t)=\infty$. We assume that there exists a function $\tau \in C^{1}\left(\left[t_{0}, \infty\right), R\right)$, such that $\tau(t) \leq \tau_{i}(t), i=0,1,2, \tau(t) \leq t$, $\tau^{\prime}(t)>0$, and $\lim _{t \rightarrow \infty} \tau(t)=\infty$.
We will consider the two cases

$$
\begin{aligned}
& \int_{t_{0}}^{\infty} \frac{1}{r^{1 / \alpha}(t)} d t=\infty, \\
& \int_{t_{0}}^{\infty} \frac{1}{r^{1 / \alpha}(t)} d t<\infty .
\end{aligned}
$$

Recently, there have been a large number of papers devoted to the oscillation of the delay differential equations; see [1-8]. Furthermore, there have been a large number of works on the oscillation of the neutral differential equations, and we refer the readers to the articles [9-25].

Agarwal and Grace [3] studied the oscillation for functional differential equations of higher order,

$$
\left(\left(x^{(n-1)}(t)\right)^{\alpha}\right)^{\prime}+q(t) f(x(g(t)))=0,
$$

and established some sufficient conditions for oscillation of (4).

Sun and Meng [6] examined the oscillation of (1), where $p(t)=0, n=2$.

$\mathrm{Xu}$ and Xia [7], by means of Riccati transformation technique, established some oscillation criteria for certain even order delay differential equations:

$$
\left(\left|(x(t))^{(n-1)}\right|^{\alpha-1}(x(t))^{(n-1)}\right)^{\prime}+F(t, x(g(t)))=0 .
$$


In 2011, Zhang et al. [8] studied the oscillatory behavior of the following higher-order half-linear delay differential equation:

$$
\left(r(t)\left(x^{(n-1)}(t)\right)^{\alpha}\right)^{\prime}+q(t) x^{\beta}(\tau(t))=0, \quad t \geq t_{0}
$$

where $\int_{t_{0}}^{\infty} r^{-(1 / \alpha)}(t) d t<\infty$.

In 2013, Zhang et al. [23] improved those reported in [8].

Han et al. [9] studied the oscillation of second-order neutral differential equations:

$$
\left(r(t) \psi(x(t))\left|Z^{\prime}(t)\right|^{\alpha-1} Z^{\prime}(t)\right)^{\prime}+q(t) f(x(\sigma(t)))=0,
$$

where $Z(t)=x(t)+p(t) x(t-\tau)$ and $\alpha>0,0 \leq p(t)<1$. Some new oscillation criteria are established for the secondorder nonlinear neutral delay differential equations:

$$
\left[r(t)[x(t)+p(t) x(\tau(t))]^{\prime}\right]^{\prime}+q(t) f(x(\sigma(t)))=0
$$

where $\int_{t_{0}}^{\infty} 1 / r(t) d t<\infty, 0 \leq p(t) \leq p_{0}<+\infty$.

Meng and $\mathrm{Xu}$ [19], by using the Riccati transformation technique and inequalities, considered the oscillation for even order quasilinear neutral differential equations:

$$
\begin{aligned}
& \left(r(t)\left|(x(t)+p(t) x(t-\sigma))^{(n-1)}\right|^{\alpha-1}\right. \\
& \left.\times(x(t)+p(t) x(t-\sigma))^{(n-1)}\right)^{\prime} \\
& +q(t) f(x(\sigma(t)))=0,
\end{aligned}
$$

where $t \geq t_{0}, 0 \leq p(t)<1$.

In 2012, Sun et al. [22] considered the oscillation criteria for even order nonlinear neutral differential equations:

$$
\left(r(t) z^{(n-1)}(t)\right)^{\prime}+q(t) f(x(\sigma(t)))=0,
$$

where $z(t)=x(t)+p(t) x(\tau(t)), n \geq 2$ is even integer, and $0 \leq p(t) \leq p_{0}<+\infty$. The results are obtained when $\int^{\infty} r^{-1}(t) d t=\infty$ or $\int^{\infty} r^{-1}(t) d t<\infty$. These criteria obtained in this paper extended and improved some known results in the literatures.

In 2013, Agarwal et al. [24] considered the oscillation criteria for even order neutral differential equations:

$$
(x(t)+p(t) x(\tau(t)))^{n}+q(t) x(\sigma(t))=0 .
$$

Some new criteria are established that improve a number of related results reported in the literature and can be used in cases where known theorems fail to apply.

In 2014, Zhang et al. [25] study oscillation and asymptotic behavior of solutions to two classes of higher-order delay damped differential equations with $p$-Laplacian like operators:

$$
\begin{aligned}
\left(a(t)\left|x^{(n-1)}(t)\right|^{\alpha-1} x^{(n-1)}(t)\right)^{\prime} & \\
+r(t)|x(t)|^{\alpha-1} x^{(n-1)}(t) & \\
+q(t)|x(g(t))|^{\alpha-1} x(g(t)) & =0, \\
t & \geq t_{0},
\end{aligned}
$$

where $\alpha>0$. Some new criteria are presented that improve the related contributions to the subject.

Clearly, the equations (4)-(12) are special cases of (1). The purpose of this paper is to extend and improve the abovementioned oscillation theorems for certain even order neutral delay differential equations with mixed nonlinearities (1).

The paper is organized as follows. In the next section, we present some lemmas which will be used in the following results. In Sections 3 and 4, by developing Riccati transformations technique and inequalities, some sufficient conditions for oscillation of all solutions of (1) are established. In Section 5, we give an example to illustrate Theorem 11.

\section{Lemmas}

In this section, in order to prove our main results, we need the following lemmas.

Lemma 1 (see [5]). Let $u \in C^{n}\left(\left[t_{0}, \infty\right), R^{+}\right)$. If $u^{(n)}(t)$ is eventually of one sign for all large $t$, then there exist $t_{x}>t_{1}$, for some $t_{1}>t_{0}$, and an integer $l, 0 \leq l \leq n$, with $n+l$ even for $u^{(n)}(t) \geq 0$ or $n+l$ odd for $u^{(n)}(t) \leq 0$ such that $l>0$ implies that $u^{(k)}(t)>0$ for $t>t_{x}, k=0,1, \ldots, l-1$, and $l \leq n-1$ implies that $(-1)^{l+k} u^{(k)}(t)>0$ for $t>t_{x}, k=l, l+1, \ldots, n-1$.

Lemma 2 (see [1], Lemma 2.2.2). If the function $u$ is as in Lemma 1 and $u^{(n-1)}(t) u^{(n)}(t) \leq 0$ for $t>t_{x}$, then, for every $\lambda, 0<\lambda<1$, there exists a constant $M>0$ such that

$$
u(\lambda t) \geq M t^{n-1}\left|u^{(n-1)}(t)\right|
$$

for all larget.

Lemma 3 (see [1], Lemma 2.2.3). If the function $u$ is as in Lemma 1 and $u^{(n-1)}(t) u^{(n)}(t) \leq 0$ for $t>t_{x}, \lim _{t \rightarrow \infty} u(t) \neq 0$, then, for every $\lambda, 0<\lambda<1$, such that

$$
u(t) \geq \frac{\lambda}{(n-1) !} t^{n-1}\left|u^{(n-1)}(t)\right|,
$$

for all larget.

Lemma 4 (see $[2,5]$ ). Consider the half-linear differential equations

$$
\left(a(t)\left|x^{\prime}(t)\right|^{\alpha-1} x^{\prime}(t)\right)^{\prime}+q(t)|x(t)|^{\alpha-1} x(t)=0,
$$


where $\alpha>0, a, q \in C\left(\left[t_{0}, \infty\right), R^{+}\right)$. Then every solution of (15) is nonoscillatory if and only if there exist a real number $T \geq t_{0}$ and a function $v \in C^{1}\left(\left[t_{0}, \infty\right), R\right)$, such that

$$
\begin{array}{r}
v^{\prime}(t)+\alpha a^{-(1 / \alpha)}(t)|\nu(t)|^{(\alpha+1) / \alpha}+q(t) \leq 0, \\
t \in[T, \infty) .
\end{array}
$$

Lemma 5. If $A$ and $B$ are nonnegative constants, then

$$
A^{\gamma}-\gamma A B^{\gamma-1}+(\gamma-1) B^{\gamma} \geq 0, \quad \gamma>1,
$$

and the equality holds if and only if $A=B$.

In the next section, by developing Riccati transformations technique and inequalities, some sufficient conditions for oscillation of all solutions of (1) are established.

\section{Oscillation Criteria for an Oscillating Function $p$}

In this section, we assume the following.

$(\mathrm{H}) p$ is an oscillating function, and $\lim _{t \rightarrow \infty} p(t)=0$.

Lemma 6. Assume that (2) holds. Furthermore, assume that $x$ is an eventually positive solution of (1), which is bounded and does not converge to zero. Then there exists $t_{1} \geq t_{0}$, such that

$$
\begin{array}{r}
z(t)>0, \quad z^{\prime}(t)>0, \quad z^{(n-1)}(t)>0, \quad z^{(n)}(t) \leq 0, \\
\forall t \geq t_{1} .
\end{array}
$$

Proof. Since $x$ is an eventually positive solution of (1), there exists a constant $t_{1} \geq t_{0}$, such that $x(t)>0, x(\sigma(t))>0$, and $x\left(\tau_{i}(t)\right)>0, i=0,1,2$, for all $t \geq t_{1}$. Then, by (1), we have $\left(r(t)\left|z^{(n-1)}(t)\right|^{\alpha-1} z^{(n-1)}(t)\right)^{\prime} \leq 0, t \geq t_{1}$.

Furthermore, since $x$ is a bounded solution and $\lim _{t \rightarrow \infty} x(t) \neq 0$, by $(\mathrm{H})$ we know that $\lim _{t \rightarrow \infty} p(t) x(\sigma(t))=0$; then there exists $t_{2} \geq t_{1}$, such that $z(t)=x(t)+p(t) x(\sigma(t))>0, t \geq t_{2}$. So $z$ is eventually positive and bounded.

The rest of the proof is similar to that of Meng and Xu [19, Lemma 2.3], so it is omitted.

Theorem 7. Assume that $(H)$ and (2) hold. Furthermore, assume that there exists a constant $\lambda, 0<\lambda<1$, and, for every constant $M>0$, assume that there exists a positive function $\rho \in C^{1}\left(\left[t_{0}, \infty\right), R\right)$, for sufficiently large $t_{1} \geq t_{0}$, such that

$$
\begin{aligned}
\limsup _{t \rightarrow \infty} \int_{t_{1}}^{t}(\rho(s) Q(s) & \\
& \left.\quad-\frac{r(s)\left(\rho^{\prime}(s)\right)^{\alpha+1}}{(\alpha+1)^{\alpha+1}\left(\lambda M \rho(s) \tau^{n-2}(s) \tau^{\prime}(s)\right)^{\alpha}}\right) d s=\infty
\end{aligned}
$$

where

$$
\begin{gathered}
Q(t):=\frac{1}{2^{\alpha}}\left\{q_{0}(t)+\left[k_{1} q_{1}(t)\right]^{1 / k_{1}}\left[k_{2} q_{2}(t)\right]^{1 / k_{2}}\right\}, \\
k_{1}:=\frac{(\gamma-\beta)}{(\gamma-\alpha)}, \quad k_{2}:=\frac{(\gamma-\beta)}{(\alpha-\beta)} .
\end{gathered}
$$

Then every bounded solution of (1) is oscillatory or converges to zero.

Proof. Suppose that (1) has a bounded nonoscillatory solution $x$. We may assume without loss of generality that there exists a number $t_{1} \geq t_{0}$, such that $x(t)>0, x(\sigma(t))>0$, and $x(\tau(t))>$ 0 , for all $t \geq t_{1}$. Furthermore, we assume that $\lim _{t \rightarrow \infty} x(t) \neq$ 0 . Using the definition of $z$ and Lemma 6 , we have $z(t)>0$, $z^{\prime}(t)>0, z^{(n-1)}(t)>0$, and $z^{(n)}(t) \leq 0, t \geq t_{1}$. Hence there exists $t_{2} \geq t_{1}$, such that

$$
x(t)=z(t)-p(t) x(\sigma(t)) \geq \frac{z(t)}{2}, \quad t \geq t_{2} .
$$

From (1) and the above inequality, we obtain

$$
\begin{aligned}
& \left(r(t)\left(z^{(n-1)}(t)\right)^{\alpha}\right)^{\prime}+\frac{1}{2^{\alpha}} q_{0}(t) z^{\alpha}(\tau(t))+\frac{1}{2^{\beta}} q_{1}(t) z^{\beta}(\tau(t)) \\
& \quad+\frac{1}{2^{\gamma}} q_{2}(t) z^{\gamma}(\tau(t)) \leq 0, \quad t \geq t_{3} \geq t_{2} .
\end{aligned}
$$

Because of $z^{\prime}(t)>0$, by Lemma $2, z^{(n-1)}(t)>0$, and $z^{(n)}(t) \leq$ 0 , there exists $t_{4} \geq t_{3}$, and, for every $0<\lambda<1$, there exists a constant $M>0$, we have

$$
z^{\prime}(\lambda \tau(t)) \geq M \tau^{n-2}(t) z^{(n-1)} \tau(t) \geq M \tau^{n-2}(t) z^{(n-1)}(t),
$$

for $t \geq t_{4}$. We define the function $\omega$ by

$$
\omega(t)=\rho(t) r(t)\left(\frac{z^{(n-1)}(t)}{z(\lambda \tau(t))}\right)^{\alpha}, \quad t \geq t_{4} .
$$

Then $\omega(t)>0, t \geq t_{4}$. Next differentiating (24), we get

$$
\begin{aligned}
\omega^{\prime}(t)= & \rho^{\prime}(t) r(t)\left(\frac{z^{(n-1)}(t)}{z(\lambda \tau(t))}\right)^{\alpha}+\rho(t) \frac{\left(r(t)\left(z^{(n-1)}(t)\right)^{\alpha}\right)^{\prime}}{z^{\alpha}(\lambda \tau(t))} \\
& -\alpha \lambda \rho(t) \frac{r(t)\left(z^{(n-1)}(t)\right)^{\alpha} \tau^{\prime}(t) z^{\prime}(\lambda \tau(t))}{z^{\alpha+1}(\lambda \tau(t))} .
\end{aligned}
$$

So by (22) and (23), we obtain

$$
\begin{aligned}
\omega^{\prime}(t) \leq & \frac{\rho^{\prime}(t)}{\rho(t)} \omega(t)-\rho(t) \\
& \times\left(\frac{1}{2^{\alpha}} q_{0}(t)+\frac{1}{2^{\beta}} q_{1}(t) z^{\beta-\alpha}(\tau(t))\right. \\
& \left.+\frac{1}{2^{\gamma}} q_{2}(t) z^{\gamma-\alpha}(\tau(t))\right)\left(\frac{z(\tau(t))}{z(\lambda \tau(t))}\right)^{\alpha} \\
& -\frac{\alpha \lambda M \tau^{n-2}(t) \tau^{\prime}(t)}{(\rho(t) r(t))^{1 / \alpha}} \omega^{(\alpha+1) / \alpha}(t) .
\end{aligned}
$$


Let

$$
a=k_{1} \frac{1}{2^{\beta}} q_{1}(t) z^{\beta-\alpha}(\tau(t)), \quad b=k_{2} \frac{1}{2^{\gamma}} q_{2}(t) z^{\gamma-\alpha}(\tau(t)),
$$

where $k_{1}$ and $k_{2}$ are defined as in Theorem 7. Using the inequality

$$
\frac{|a|}{p}+\frac{|b|}{q} \geq|a|^{1 / p}|b|^{1 / q}, \quad p>1, \quad q>1, \quad \frac{1}{p}+\frac{1}{q}=1,
$$

we have

$$
\begin{aligned}
& \frac{1}{2^{\beta}} q_{1}(t) z^{\beta-\alpha}(\tau(t))+\frac{1}{2^{\gamma}} q_{2}(t) z^{\gamma-\alpha}(\tau(t)) \\
& \geq\left[k_{1} \frac{1}{2^{\beta}} q_{1}(t) z^{\beta-\alpha}(\tau(t))\right]^{1 / k_{1}}\left[k_{2} \frac{1}{2^{\gamma}} q_{2}(t) z^{\gamma-\alpha}(\tau(t))\right]^{1 / k_{2}} \\
& \quad=\frac{1}{2^{\alpha}}\left[k_{1} q_{1}(t)\right]^{1 / k_{1}}\left[k_{2} q_{2}(t)\right]^{1 / k_{2}},
\end{aligned}
$$

so we get

$$
\begin{aligned}
\omega^{\prime}(t) \leq & \frac{\rho^{\prime}(t)}{\rho(t)} \omega(t)-\rho(t) Q(t) \\
& -\frac{\alpha \lambda M \tau^{n-2}(t) \tau^{\prime}(t)}{(\rho(t) r(t))^{1 / \alpha}} \omega^{(\alpha+1) / \alpha}(t) .
\end{aligned}
$$

Let

$$
\begin{aligned}
& A=\left(\alpha \lambda M \frac{\tau^{n-2}(t) \tau^{\prime}(t)}{(\rho(t) r(t))^{1 / \alpha}}\right)^{1 / \gamma} \omega(t), \\
& B=\left(\frac{\rho^{\prime}(t)}{\gamma \rho(t)}\left(\alpha \lambda M \frac{\tau^{n-2}(t) \tau^{\prime}(t)}{(\rho(t) r(t))^{1 / \alpha}}\right)^{-(1 / \gamma)}\right)^{1 /(\gamma-1)},
\end{aligned}
$$

where $\gamma=(\alpha+1) / \alpha>1$. Applying the inequality in Lemma 5, we obtain

$$
\begin{gathered}
\frac{\rho^{\prime}(t)}{\rho(t)} \omega(t)-\alpha \lambda M \frac{\tau^{n-2}(t) \tau^{\prime}(t)}{(\rho(t) r(t))^{1 / \alpha}} \omega^{\gamma}(t) \\
\leq \frac{r(t)\left(\rho^{\prime}(t)\right)^{\alpha+1}}{(\alpha+1)^{\alpha+1}\left(\lambda M \rho(t) \tau^{n-2}(t) \tau^{\prime}(t)\right)^{\alpha}} .
\end{gathered}
$$

Thus, by (30) and (32), we get

$$
\begin{aligned}
\omega^{\prime}(t) \leq- & (\rho(t) Q(t) \\
& \left.-\frac{r(t)\left(\rho^{\prime}(t)\right)^{\alpha+1}}{(\alpha+1)^{\alpha+1}\left(\lambda M \rho(t) \tau^{n-2}(t) \tau^{\prime}(t)\right)^{\alpha}}\right) .
\end{aligned}
$$

Integrating (33) from $t_{1}$ to $t$, we have

$$
\begin{aligned}
\omega(t) \leq & \omega\left(t_{1}\right) \\
& -\int_{t_{1}}^{t}(\rho(s) Q(s) \\
& \left.\quad-\frac{r(s)\left(\rho^{\prime}(s)\right)^{\alpha+1}}{(\alpha+1)^{\alpha+1}\left(\lambda M \rho(s) \tau^{n-2}(s) \tau^{\prime}(s)\right)^{\alpha}}\right) d s .
\end{aligned}
$$

Let $t \rightarrow \infty$ in (34), which leads to a contradiction with (19). The proof is complete.

Theorem 8. Assume that $(H)$ and (2) hold, and there exists a constant $\lambda, 0<\lambda<1$, and, for every constant $M>0$, such that

$$
\begin{gathered}
\left(\left(\frac{(r(t))^{1 / \alpha}}{\lambda M \tau^{n-2}(t) \tau^{\prime}(t)}\right)^{\alpha}\left|x^{\prime}(t)\right|^{\alpha-1} x^{\prime}(t)\right)^{\prime} \\
+Q(t)|x(t)|^{\alpha-1} x(t)=0,
\end{gathered}
$$

is oscillatory, where $Q$ is defined as in Theorem 7. Then every bounded solution of (1) is oscillatory or converges to zero.

Proof. Suppose that (1) has a bounded nonoscillatory solution $x$. We may assume without loss of generality that there exists $t_{1} \geq t_{0}$, such that $x(t)>0, x(\sigma(t))>0$, and $x(\tau(t))>0$, for all $t \geq t_{1}$. Furthermore, we assume that $\lim _{t \rightarrow \infty} x(t) \neq 0$. We define $v$ by

$$
\nu(t)=r(t)\left(\frac{z^{(n-1)}(t)}{z(\lambda \tau(t))}\right)^{\alpha} .
$$

Proceeding as in the proof of Theorem 7 , for every $0<\lambda<1$, there exists $M>0$, and we have

$$
\nu^{\prime}(t)+Q(\mathrm{t})+\frac{\alpha \lambda M \tau^{n-2}(t) \tau^{\prime}(t)}{(r(t))^{1 / \alpha}} v^{(\alpha+1) / \alpha}(t) \leq 0 .
$$

That is,

$$
\begin{aligned}
& \nu^{\prime}(t)+Q(t) \\
& \quad+\alpha\left[\left(\frac{(r(t))^{1 / \alpha}}{\lambda M \tau^{n-2}(t) \tau^{\prime}(t)}\right)^{\alpha}\right]^{-(1 / \alpha)} \nu^{(\alpha+1) / \alpha}(t) \leq 0 .
\end{aligned}
$$

Based on Lemma 4, we obtain that (35) is nonoscillatory, which leads to a contradiction. The proof is complete.

Theorem 9. Assume that $(H)$ and (3) hold. Furthermore, assume that there exists a constant $\lambda, 0<\lambda<1$, and, for every constant $M>0$, assume that there exists a positive function $\rho \in C^{1}\left(\left[t_{0}, \infty\right), R\right)$, such that (19) holds. If, for sufficiently large $t_{1} \geq t_{0}$,

$$
\begin{aligned}
\limsup _{t \rightarrow \infty} \int_{t_{1}}^{t} & {\left[\left(\frac{\lambda}{(n-2) !}\right)^{\alpha} Q(s) \tau^{\alpha(n-2)}(s) \delta^{\alpha}(s)\right.} \\
& \left.-\left(\frac{\alpha}{\alpha+1}\right)^{\alpha+1} \frac{1}{r^{1 / \alpha}(s) \delta(s)}\right] d s=\infty,
\end{aligned}
$$


where $Q$ and $h$ are defined as in Theorem 7 , and $\delta(t)=$ $\int_{t}^{\infty}\left(1 / r^{1 / \alpha}(s)\right) d s$, then every bounded solution of (1) is oscillatory or converges to zero.

Proof. Suppose that (1) has a bounded nonoscillatory solution $x$. We may assume without loss of generality that there exists $t_{1} \geq t_{0}$, such that $x(t)>0, x(\sigma(t))>0$, and $x(\tau(t))>0$, for all $t \geq t_{1}$. Then it follows from (1) that

$$
\left(r(t)\left|z^{(n-1)}(t)\right|^{\alpha-1} z^{(n-1)}(t)\right)^{\prime} \leq 0, \quad t \geq t_{1} .
$$

Therefore, $r(t)\left|z^{(n-1)}(t)\right|^{\alpha-1} z^{(n-1)}(t)$ is a nonincreasing function on $\left[t_{1}, \infty\right)$. Consequently, it is easy to conclude that there exist two possible cases of the sign of $z^{(n-1)}(t)$. Furthermore, we assume that $\lim _{t \rightarrow \infty} x(t) \neq 0$.

Case I. If $z^{(n-1)}(t)>0$, for $t \geq t_{1}$, then we go back to the proof of Theorem 7, and we get a contradiction to (19), so we omit the details.

Case II. $z^{(n-1)}(t)<0$, for $t \geq t_{1}$. Applying Lemma 1 , we get $z^{(n-2)}(t)>0$. Define the function $v$ by

$$
v(t)=\frac{r(t)\left(-z^{(n-1)}(t)\right)^{\alpha-1} z^{(n-1)}(t)}{\left(z^{(n-2)}(t)\right)^{\alpha}}, \quad t \geq t_{1} .
$$

Then $v(t)<0$ for $t \geq t_{1}$. Noting that $r(t)\left|z^{(n-1)}(t)\right|^{\alpha-1} z^{(n-1)}(t)$ is nonincreasing, we obtain

$$
r^{1 / \alpha}(s) z^{(n-1)}(s) \leq r^{1 / \alpha}(t) z^{(n-1)}(t), \quad s \geq t .
$$

Dividing (42) by $r^{1 / \alpha}(s)$ and integrating it from $t$ to $l \quad(l \geq t)$, we have

$$
z^{(n-2)}(l) \leq z^{(n-2)}(t)+r^{1 / \alpha}(t) z^{(n-1)}(t) \int_{t}^{l} \frac{1}{r^{1 / \alpha}(s)} d s .
$$

Letting $l \rightarrow \infty$ in the above inequality, we get

$$
0 \leq z^{(n-2)}(t)+r^{1 / \alpha}(t) z^{(n-1)}(t) \delta(t)
$$

which implies that

$$
-1 \leq \frac{r^{1 / \alpha}(t) z^{(n-1)}(t)}{z^{(n-2)}(t)} \delta(t), \quad t \geq t_{1},
$$

where $\delta$ is defined as in Theorem 9. Hence, by (41), we obtain

$$
-1 \leq v(t) \delta^{\alpha}(t) \leq 0, \quad t \geq t_{1} .
$$

Differentiating (41), we have

$$
\begin{aligned}
v^{\prime}(t)= & \frac{\left(r(t)\left(-z^{(n-1)}(t)\right)^{\alpha-1} z^{(n-1)}(t)\right)^{\prime}}{\left(z^{(n-2)}(t)\right)^{\alpha}} \\
& -\frac{\alpha r(t)\left(-z^{(n-1)}(t)\right)^{\alpha-1} z^{(n-1)}(t) z^{(n-1)}(t)}{\left(z^{(n-2)}(t)\right)^{\alpha+1}} .
\end{aligned}
$$

From (22), we get

$$
\begin{aligned}
v^{\prime}(t) \leq & {\left[-\frac{1}{2^{\alpha}} q_{0}(t) z^{\alpha}(\tau(t))-\frac{1}{2^{\beta}} q_{1}(t) z^{\beta}(\tau(t))\right.} \\
& \left.-\frac{1}{2^{\gamma}} q_{2}(t) z^{\gamma}(\tau(t))\right] \frac{1}{\left(z^{(n-2)}(t)\right)^{\alpha}} \\
& -\frac{\alpha r(t)\left(-z^{(n-1)}(t)\right)^{\alpha-1} z^{(n-1)}(t) z^{(n-1)}(t)}{\left(z^{(n-2)}(t)\right)^{\alpha+1}} .
\end{aligned}
$$

On the other hand, by $\lim _{t \rightarrow \infty} z(t) \neq 0$ and Lemma 3, we obtain

$$
z(t) \geq \frac{\lambda}{(n-2) !} t^{n-2} z^{(n-2)}(t)
$$

that is, because of $z^{(n-1)}<0$,

$$
\begin{aligned}
z(\tau(t)) & \geq \frac{\lambda}{(n-2) !} \tau^{n-2}(t) z^{(n-2)}(\tau(t)) \\
& \geq \frac{\lambda}{(n-2) !} \tau^{n-2}(t) z^{(n-2)}(t),
\end{aligned}
$$

for every $0<\lambda<1$ and $t \geq t_{1}$. Then from (41), (48), and (50), we have

$$
\begin{aligned}
v^{\prime}(t) \leq & {\left[-\frac{1}{2^{\alpha}} q_{0}(t)-\frac{1}{2^{\beta}} q_{1}(t) z^{\beta-\alpha}(\tau(t))\right.} \\
& \left.-\frac{1}{2^{\gamma}} q_{2}(t) z^{\gamma-\alpha}(\tau(t))\right] \frac{z^{\alpha}(\tau(t))}{\left(z^{(n-2)}(t)\right)^{\alpha}} \\
& -\frac{\alpha r(t)\left(-z^{(n-1)}(t)\right)^{\alpha-1} z^{(n-1)}(t) z^{(n-1)}(t)}{\left(z^{(n-2)}(t)\right)^{\alpha+1}} \\
\leq & -\left(\frac{\lambda}{(n-1) !}\right)^{\alpha} Q(t) \tau^{\alpha(n-2)}(t) \\
& -\frac{\alpha}{r^{1 / \alpha}(t)}(-v(t))^{(\alpha+1) / \alpha}
\end{aligned}
$$

where $Q$ is defined as in Theorem 7. Multiplying (51) by $\delta^{\alpha}(t)$ and integrating it from $t_{1}$ to $t$, we get

$$
\begin{gathered}
\delta^{\alpha}(t) v(t)-\delta^{\alpha}\left(t_{1}\right) v\left(t_{1}\right)+\alpha \int_{t_{1}}^{t} \frac{\delta^{\alpha-1}(s)}{r^{1 / \alpha}(s)} v(s) d s \\
+\left(\frac{\lambda}{(n-2) !}\right)^{\alpha} \int_{t_{1}}^{t} Q(s) \tau^{\alpha(n-2)}(s) \delta^{\alpha}(s) d s \\
+\alpha \int_{t_{1}}^{t} \frac{\delta^{\alpha}(s)}{r^{1 / \alpha}(s)}(-v(s))^{(\alpha+1) / \alpha} d s \leq 0 .
\end{gathered}
$$

Let

$$
\begin{aligned}
& A=\left(\frac{\alpha \delta^{\alpha}(s)}{r^{1 / \alpha}(s)}\right)^{1 / \gamma}(-v(s)), \\
& B=\left(\frac{\alpha^{2}}{\alpha+1} \frac{\delta^{\alpha-1}(s)}{r^{1 / \alpha}(s)}\left(\frac{\alpha \delta^{\alpha}(s)}{r^{1 / \alpha}(s)}\right)^{-(1 / \gamma)}\right)^{1 /(\gamma-1)},
\end{aligned}
$$


where $\gamma=(\alpha+1) / \alpha>1$. Applying the inequality in Lemma 5 , we obtain

$$
\begin{aligned}
& \frac{\alpha \delta^{\alpha-1}(s)}{r^{1 / \alpha}(s)}(-v(s))-\frac{\alpha \delta^{\alpha}(s)}{r^{1 / \alpha}(s)}(-v(s))^{(\alpha+1) / \alpha} \\
& \leq\left(\frac{\alpha}{\alpha+1}\right)^{\alpha+1} \frac{1}{\mathrm{r}^{1 / \alpha}(s) \delta(s)} .
\end{aligned}
$$

Therefore, it follows from (52) that

$$
\begin{gathered}
\delta^{\alpha}(t) v(t)+\int_{t_{1}}^{t}\left[\left(\frac{\lambda}{(n-2) !}\right)^{\alpha} \mathrm{Q}(s) \tau^{\alpha(n-2)}(s) \delta^{\alpha}(s)\right. \\
\left.\quad-\left(\frac{\alpha}{\alpha+1}\right)^{\alpha+1} \frac{1}{r^{1 / \alpha}(s) \delta(s)}\right] d s \\
\leq \delta^{\alpha}\left(t_{1}\right) v\left(t_{1}\right) .
\end{gathered}
$$

From (39) and the above inequality, we get a contradiction to (46). The proof is complete.

Theorem 10. Assume that $(H)$ and (3) hold. Furthermore, assume that there exists a constant $\lambda, 0<\lambda<1$, and for every constant $M>0$ such that (35) is oscillatory. If, for sufficiently large $t_{1} \geq t_{0}$, one has (39), where $Q$ and $h$ are defined as in Theorem 7 and $\delta$ is defined as in Theorem 9, then every bounded solution of (1) is oscillatory or converges to zero.

\section{Oscillation Criteria for $0 \leq p(t)<1$}

In this section, we assume that $0 \leq p(t)<1$.

Theorem 11. Assume that (2) holds, there exists a constant $\lambda$, $0<\lambda<1$, and, for every constant $M>0$, there exists a positive function $\rho \in C^{1}\left(\left[t_{0}, \infty\right), R\right)$, such that, for sufficiently large $t_{1} \geq$ $t_{0}$,

$$
\begin{aligned}
\limsup _{t \rightarrow \infty} \int_{t_{1}}^{t} & (\rho(s) \overline{Q(s)} \\
& \left.\quad-\frac{r(s)\left(\rho^{\prime}(s)\right)^{\alpha+1}}{(\alpha+1)^{\alpha+1}\left(\lambda M \rho(s) \tau^{n-2}(s) \tau^{\prime}(s)\right)^{\alpha}}\right) d s=\infty,
\end{aligned}
$$

where

$$
\begin{aligned}
& \overline{Q(t)}=\xi^{\alpha}(t)\left\{q_{0}(t)+\left[k_{1} q_{1}(t)\right]^{1 / k_{1}}\left[k_{2} q_{2}(t)\right]^{1 / k_{2}}\right\}, \\
& \xi(t)=\min \left\{1-p\left(\tau_{0}(t)\right), 1-p\left(\tau_{1}(t)\right), 1-p\left(\tau_{2}(t)\right)\right\},
\end{aligned}
$$

and $k_{1}$ and $k_{2}$ are defined as in Theorem 7 . Then every solution of (1) is oscillatory.

Proof. Suppose that (1) has a nonoscillatory solution $x$. Without loss of generality, we may assume that there exists $t_{1} \geq t_{0}$, such that $x(t)>0, x(\sigma(t))>0$, and $x(\tau(t))>0$, for all $t \geq t_{1}$. Similar to the proof of Lemma 2.3 in [19], there exists $t_{2} \geq t_{1}$, such that

$z(t)>0, \quad z^{\prime}(t)>0, \quad z^{(n-1)}(t)>0, \quad z^{(n)}(t) \leq 0$,

From the definition of $z$, we have

$$
\begin{aligned}
x(t) & =z(t)-p(t) x(\sigma(t)) \geq z(t)-p(t) z(\sigma(t)) \\
& \geq(1-p(t)) z(t), \quad t \geq t_{3} \geq t_{2} .
\end{aligned}
$$

Since $\lim _{t \rightarrow \infty} \tau(t)=\infty$, there exists $t_{4} \geq t_{3}$, such that $\tau(t) \geq$ $t_{4}, t \geq t_{4}$, so

$$
x(\tau(t)) \geq(1-p(\tau(t))) z(\tau(t)), \quad t \geq t_{4} .
$$

From (1), (59), and (60), we get

$$
\begin{gathered}
\left(r(t)\left(z^{(n-1)}(t)\right)^{\alpha}\right)^{\prime}+1-p\left(\tau_{0}(t)\right)^{\alpha} q_{0}(t) z^{\alpha}(\tau(t)) \\
+1-p\left(\tau_{1}(t)\right)^{\beta} q_{1}(t) z^{\beta}(\tau(t)) \\
+1-p\left(\tau_{2}(t)\right)^{\gamma} q_{2}(t) z^{\gamma}(\tau(t)) \leq 0 \\
t \geq t_{4} .
\end{gathered}
$$

For every $0<\lambda<1$, we define the function

$$
\omega(t)=\rho(t) r(t)\left(\frac{z^{(n-1)}(t)}{z(\lambda \tau(t))}\right)^{\alpha}, \quad t \geq t_{4} .
$$

Then $\omega(t)>0, t \geq t_{4}$. Next differentiating (62), we obtain

$$
\begin{aligned}
\omega^{\prime}(t)= & \rho^{\prime}(t) r(t)\left(\frac{z^{(n-1)}(t)}{z(\lambda \tau(t))}\right)^{\alpha}+\rho(t) \frac{\left(r(t)\left(z^{(n-1)}(t)\right)^{\alpha}\right)^{\prime}}{z^{\alpha}(\lambda \tau(t))} \\
& -\alpha \lambda \rho(t) r(t) \frac{\left(z^{(n-1)}(t)\right)^{\alpha} z^{\prime}(\lambda \tau(t))}{z^{\alpha+1}(\lambda \tau(t))} .
\end{aligned}
$$

From (23), (61), and (62), we have

$$
\begin{aligned}
\omega^{\prime}(t) \leq & \frac{\rho^{\prime}(t)}{\rho(t)} \omega(t)-\rho(t) \\
& \times\left(\xi^{\alpha}(t) q_{0}(t)+\xi^{\beta}(t) q_{1}(t) z^{\beta-\alpha}(\tau(t))\right. \\
& \left.\quad+\xi^{\gamma}(t) q_{2}(t) z^{\gamma-\alpha}(\tau(t))\right)\left(\frac{z(\tau(t))}{z(\lambda \tau(t))}\right)^{\alpha} \\
& -\alpha \lambda M \frac{\tau^{n-2}(t) \tau^{\prime}(t)}{(\rho(t) r(t))^{1 / \alpha}} \omega^{(\alpha+1) / \alpha}(t),
\end{aligned}
$$

where $\xi$ is defined as in Theorem 11. Setting

$$
\begin{aligned}
& a=k_{1} \xi^{\beta}(t) q_{1}(t) z^{\beta-\alpha}(\tau(t)), \\
& b=k_{2} \xi^{\gamma}(t) q_{2}(t) z^{\gamma-\alpha}(\tau(t)),
\end{aligned}
$$


by inequality (28), we get

$$
\begin{aligned}
\xi^{\beta}(t) & q_{1}(t) z^{\beta-\alpha}(\tau(t))+\xi^{\gamma}(t) q_{2}(t) z^{\gamma-\alpha}(\tau(t)) \\
\geq & {\left[k_{1} \xi^{\beta}(t) q_{1}(t) z^{\beta-\alpha}(\tau(t))\right]^{1 / k_{1}} } \\
& \times\left[k_{2} \xi^{\gamma}(t) q_{2}(t) z^{\gamma-\alpha}(\tau(t))\right]^{1 / k_{2}} \\
= & \xi^{\alpha}(t)\left[k_{1} q_{1}(t)\right]^{1 / k_{1}}\left[k_{2} q_{2}(t)\right]^{1 / k_{2}} ;
\end{aligned}
$$

hence,

$$
\begin{aligned}
\omega^{\prime}(t) \leq & \frac{\rho^{\prime}(t)}{\rho(t)} \omega(t)-\rho(t) \overline{Q(t)} \\
& -\alpha \lambda M \frac{\tau^{n-2}(t) \tau^{\prime}(t)}{(\rho(t) r(t))^{1 / \alpha}} \omega^{(\alpha+1) / \alpha}(t) .
\end{aligned}
$$

Let

$$
\begin{aligned}
& A=\left(\alpha \lambda M \frac{\tau^{n-2}(t) \tau^{\prime}(t)}{(\rho(t) r(t))^{1 / \alpha}}\right)^{1 / \gamma} \omega(t) \\
& B=\left(\frac{\rho^{\prime}(t)}{\gamma \rho(t)}\left(\alpha \lambda M \frac{\tau^{n-2}(t) \tau^{\prime}(t)}{(\rho(t) r(t))^{1 / \alpha}}\right)^{-(1 / \gamma)}\right)^{1 /(\gamma-1)},
\end{aligned}
$$

where $\gamma=(\alpha+1) / \alpha>1$. Applying the inequality in Lemma 5, we obtain

$$
\begin{aligned}
& \frac{\rho^{\prime}(t)}{\rho(t)} \omega(t)-\alpha \lambda M \frac{\tau^{n-2}(t) \tau^{\prime}(t)}{(\rho(t) r(t))^{1 / \alpha}} \omega^{\gamma}(t) \\
& \quad \leq \frac{r(t)\left(\rho^{\prime}(t)\right)^{\alpha+1}}{(\alpha+1)^{\alpha+1}\left(\lambda M \rho(t) \tau^{n-2}(t) \tau^{\prime}(t)\right)^{\alpha}} .
\end{aligned}
$$

Thus, by (67) and (69), we get

$$
\begin{aligned}
\omega^{\prime}(t) \leq-(\rho(t) \overline{Q(t)} & \\
& \left.-\frac{r(t)\left(\rho^{\prime}(t)\right)^{\alpha+1}}{(\alpha+1)^{\alpha+1}\left(\lambda M \rho(t) \tau^{n-2}(t) \tau^{\prime}(t)\right)^{\alpha}}\right) .
\end{aligned}
$$

Integrating (70) from $t_{1}$ to $t$, we have

$$
\begin{aligned}
\omega(t) \leq \omega\left(t_{1}\right) & \\
& -\int_{t_{1}}^{t}(\rho(s) \overline{Q(s)} \\
& \left.\quad-\frac{r(s)\left(\rho^{\prime}(s)\right)^{\alpha+1}}{(\alpha+1)^{\alpha+1}\left(\lambda M \rho(s) \tau^{n-2}(s) \tau^{\prime}(s)\right)^{\alpha}}\right) d s .
\end{aligned}
$$

Letting $t \rightarrow \infty$ in (71), we get a contradiction with (56). This completes the proof of Theorem 11.
Remark 12. From Theorem 11, we can obtain different conditions for oscillation of all solutions of (1) with different choices of $\rho$.

Theorem 13. Assume that (3) holds, assume that there exists a constant $\lambda, 0<\lambda<1$, and, for every constant $M>0$, there exists a positive function $\rho \in C^{1}\left(\left[t_{0}, \infty\right), R\right)$, such that, for sufficiently large $t_{1} \geq t_{0}$, (56) holds. If there exists a positive function $\eta \in C^{1}\left(\left[t_{0}, \infty\right), R\right), \eta^{\prime}(t) \geq 0$, such that

$$
\begin{gathered}
\int_{t_{1}}^{\infty}\left(\frac{1}{\eta(t) r(t)} \int_{t_{1}}^{t}\left(\frac{\lambda M}{(n-2) !} \tau^{n-2}(s) \delta(\tau(s))\right)^{\alpha}\right. \\
\times \eta(s) \overline{Q(s)} d s)^{1 / \alpha} d t=\infty, \\
\int_{t_{1}}^{\infty}\left(\frac{1}{\eta(t) r(t)} \int_{t_{1}}^{t}\left(\frac{\lambda M}{(n-2) !} s^{n-2} \delta(s)\right)^{\alpha}\right. \\
\times \eta(s) \overline{Q(s)} d s)^{1 / \alpha} d t=\infty,
\end{gathered}
$$

where $\delta$ is defined as in Theorem 9 and $\bar{Q}$ is defined as in Theorem 11, then every solution of (1) is oscillatory or converges to zero.

Proof. Suppose that (1) has a nonoscillatory solution $x$. We may assume without loss of generality that there exists $t_{1} \geq$ $t_{0}$, such that $x(t)>0, x(\sigma(t))>0, x(\tau(t))>0$, and $z(t)>0$, for all $t \geq t_{1}$. Furthermore, we assume that $\lim _{t \rightarrow \infty} x(t) \neq 0$. Similar to the proof of Theorem 9, we find that $r(t)\left|z^{(n-1)}(t)\right|^{\alpha-1} z^{(n-1)}(t)$ is a nonincreasing function on $\left[t_{1}, \infty\right)$ and there exist two possible cases of the sign of $z^{(n-1)}(t)$.

Case I. If $z^{(n-1)}(t)>0$, for $t \geq t_{1}$, then we go back to the proof of Theorem 11, and we get a contradiction to (56), so we omit the details.

Case II. $z^{(n-1)}(t)<0$, for $t \geq t_{1}$. Applying Lemma 1, we get $z^{(n-2)}(t)>0, z^{\prime}(t)>0$ or $z^{(n-2)}(t)>0, z^{\prime}(t)<0$.

$$
\text { If } z^{(n-2)}(t)>0, z^{\prime}(t)>0 \text {. Since } r(t)\left|z^{(n-1)}(t)\right|^{\alpha-1} z^{(n-1)}(t)
$$
is nonincreasing, we obtain

$$
\begin{aligned}
& r(s)\left|z^{(n-1)}(s)\right|^{\alpha-1} z^{(n-1)}(s) \\
& \quad \leq r(t)\left|z^{(n-1)}(t)\right|^{\alpha-1} z^{(n-1)}(t) \\
& \quad \leq r\left(t_{1}\right)\left|z^{(n-1)}\left(t_{1}\right)\right|^{\alpha-1} z^{(n-1)}\left(t_{1}\right),
\end{aligned}
$$

for $s \geq t \geq t_{1}$; that is,

$$
-z^{(n-1)}(s) \geq\left(\frac{r(t)}{r(s)}\right)^{1 / \alpha}\left(-z^{(n-1)}(t)\right) .
$$


Integrating (75) from $t$ to $\infty$, we get

$$
\begin{array}{r}
z^{(n-2)}(t) \geq r^{1 / \alpha}(t)\left(-z^{(n-1)}(t)\right) \int_{t}^{\infty} \frac{1}{r^{1 / \alpha}(s)} d s \geq M \delta(t) \\
t \geq t_{1}
\end{array}
$$

where $M=r^{1 / \alpha}\left(t_{1}\right)\left(-z^{(n-1)}\left(t_{1}\right)\right)$. From (49) and the above inequality, we obtain

$$
z(t) \geq M \frac{\lambda}{(n-2) !} t^{n-2} \delta(t)
$$

Using (59) and (77) in (1) and noting that $z^{\prime}(t)>0$, we have

$$
\begin{aligned}
- & \left(r(t)\left|z^{(n-1)}(t)\right|^{\alpha-1} z^{(n-1)}(t)\right)^{\prime} \\
= & q_{0}(t) x^{\alpha}\left(\tau_{0}(t)\right)+q_{1}(t) x^{\beta}\left(\tau_{1}(t)\right)+q_{2}(t) x^{\gamma}\left(\tau_{2}(t)\right) \\
\geq & q_{0}(t)\left(1-p\left(\tau_{0}(t)\right)\right)^{\alpha} z^{\alpha}\left(\tau_{0}(t)\right) \\
& +q_{1}(t)\left(1-p\left(\tau_{1}(t)\right)\right)^{\beta} z^{\beta}\left(\tau_{1}(t)\right) \\
& +q_{2}(t)\left(1-p\left(\tau_{2}(t)\right)\right)^{\gamma} z^{\gamma}\left(\tau_{2}(t)\right) \\
\geq & \xi^{\alpha}(t) q_{0}(t) z^{\alpha}(\tau(t))+\xi^{\beta}(t) q_{1}(t) z^{\beta}(\tau(t)) \\
& +\xi^{\gamma}(t) q_{2}(t) z^{\gamma}(\tau(t))
\end{aligned}
$$

Setting

$$
\begin{aligned}
& a=k_{1} \xi^{\beta}(t) q_{1}(t) z^{\beta}(\tau(t)), \\
& b=k_{2} \xi^{\gamma}(t) q_{2}(t) z^{\gamma}(\tau(t)),
\end{aligned}
$$

by inequality (28), we get

$$
\begin{aligned}
& \xi^{\beta}(t) q_{1}(t) z^{\beta}(\tau(t))+\xi^{\gamma}(t) q_{2}(t) z^{\gamma}(\tau(t)) \\
& \quad \geq\left[k_{1} \xi^{\beta} q_{1}(t) z^{\beta}(\tau(t))\right]^{1 / k_{1}}\left[k_{2} \xi^{\gamma} q_{2}(t) z^{\gamma}(\tau(t))\right]^{1 / k_{2}} \\
& \quad \geq \xi^{\alpha}(t)\left[k_{1} q_{1}(t)\right]^{1 / k_{1}}\left[k_{2} q_{2}(t)\right]^{1 / k_{2}} z^{\alpha}(\tau(t))
\end{aligned}
$$

Therefore, combining (77), (78), and (80), we obtain

$$
\begin{aligned}
- & \left(r(t)\left|z^{(n-1)}(t)\right|^{\alpha-1} z^{(n-1)}(t)\right)^{\prime} \\
& \geq \overline{Q(t)} z^{\alpha}(\tau(t)) \geq\left(\frac{\lambda M}{(n-2) !} \tau^{n-2}(t) \delta(\tau(t))\right)^{\alpha} \overline{Q(t)} .
\end{aligned}
$$

Define the function $u$ by

$$
\begin{aligned}
u(t) & =\eta(t) r(t)\left|z^{(n-1)}(t)\right|^{\alpha-1} z^{(n-1)}(t) \\
& =-\eta(t) r(t)\left(-z^{(n-1)}(t)\right)^{\alpha}, \quad t \geq t_{1} .
\end{aligned}
$$

Then $u(t)<0$. Differentiating $u(t)$ and from (81), we find that

$$
\begin{aligned}
u^{\prime}(t)= & -\eta^{\prime}(t) r(t)\left(-z^{(n-1)}(t)\right)^{\alpha} \\
& -\eta(t)\left(r(t)\left(-z^{(n-1)}(t)\right)^{\alpha}\right)^{\prime} \\
\leq & -\eta(t)\left(q_{0}(t) x^{\alpha}\left(\tau_{0}(t)\right)+q_{1}(t) x^{\beta}\left(\tau_{1}(t)\right)\right. \\
\quad & \left.+q_{2}(t) x^{\gamma}\left(\tau_{2}(t)\right)\right) \\
\leq & -\left(\frac{\lambda M}{(n-2) !} \tau^{n-2}(t) \delta(\tau(t))\right)^{\alpha} \eta(t) \overline{Q(t)}
\end{aligned}
$$

Integrating (83) from $t_{1}$ to $t$, we get

$$
\begin{aligned}
u(t) & -u\left(t_{1}\right) \\
\leq & -\int_{t_{1}}^{t}\left(\frac{\lambda M}{(n-2) !} \tau^{n-2}(s) \delta(\tau(s))\right)^{\alpha} \eta(s) \overline{Q(s)} d s .
\end{aligned}
$$

Therefore,

$$
\begin{aligned}
& -\eta(t) r(t)\left(-z^{(n-1)}(t)\right)^{\alpha} \\
& \leq-\int_{t_{1}}^{t}\left(\frac{\lambda M}{(n-2) !} \tau^{n-2}(s) \delta(\tau(s))\right)^{\alpha} \eta(s) \overline{Q(s)} d s
\end{aligned}
$$

that is,

$$
\begin{aligned}
& z^{(n-1)}(t) \\
& \leq-\left(\frac{1}{\eta(t) r(t)} \int_{t_{1}}^{t}\left(\frac{\lambda M}{(n-2) !} \tau^{n-2}(s) \delta(\tau(s))\right)^{\alpha}\right. \\
& \times \eta(s) \overline{Q(s)} d s)^{1 / \alpha} .
\end{aligned}
$$

Integrating the above inequality from $t_{1}$ to $l\left(l>t_{1}\right)$, we obtain

$$
\begin{gathered}
z^{(n-2)}(l)-z^{(n-2)}\left(t_{1}\right) \\
\leq-\int_{t_{1}}^{l}\left(\frac{1}{\eta(t) r(t)} \int_{t_{1}}^{t}\left(\frac{\lambda M}{(n-2) !} \tau^{n-2}(s) \delta(\tau(s))\right)^{\alpha}\right. \\
\times \eta(s) \overline{Q(s)} d s)^{1 / \alpha} d t
\end{gathered}
$$

Letting $l \rightarrow \infty$ and using (73) in (97), we have $\lim _{l \rightarrow \infty} z^{(n-2)}(l)=-\infty$, which is a contradiction with the fact that $z^{(n-2)}(t)>0$.

$$
\text { If } z^{(n-2)}(t)>0, z^{\prime}(t)<0 \text {. Because of } \lim _{t \rightarrow \infty} x(t) \neq 0,
$$
$\lim _{t \rightarrow \infty} z(t) \neq 0$. By Lemma 3, we obtain (49). Proceeding as 
in the proof of the above, (77) holds. Using (59) and (77) in (1) and noting that $z^{\prime}(t)<0$, we have

$$
\begin{aligned}
- & \left(r(t)\left|z^{(n-1)}(t)\right|^{\alpha-1} z^{(n-1)}(t)\right)^{\prime} \\
= & q_{0}(t) x^{\alpha}\left(\tau_{0}(t)\right)+q_{1}(t) x^{\beta}\left(\tau_{1}(t)\right)+q_{2}(t) x^{\gamma}\left(\tau_{2}(t)\right) \\
\geq & q_{0}(t)\left(1-p\left(\tau_{0}(t)\right)\right)^{\alpha} z^{\alpha}\left(\tau_{0}(t)\right) \\
& +q_{1}(t)\left(1-p\left(\tau_{1}(t)\right)\right)^{\beta} z^{\beta}\left(\tau_{1}(t)\right) \\
& +q_{2}(t)\left(1-p\left(\tau_{2}(t)\right)\right)^{\gamma} z^{\gamma}\left(\tau_{2}(t)\right) \\
\geq & \xi^{\alpha}(t) q_{0}(t) z^{\alpha}(t)+\xi^{\beta}(t) q_{1}(t) z^{\beta}(t) \\
& +\xi^{\gamma}(t) q_{2}(t) z^{\gamma}(t)
\end{aligned}
$$

Setting

$$
\begin{aligned}
& a_{1}=k_{1} \xi^{\beta}(t) q_{1}(t) z^{\beta}(t), \\
& b_{1}=k_{2} \xi^{\gamma}(t) q_{2}(t) z^{\gamma}(t),
\end{aligned}
$$

by inequality (28), we get

$$
\begin{aligned}
& \xi^{\beta}(t) q_{1}(t) z^{\beta}(t)+\xi^{\gamma}(t) q_{2}(t) z^{\gamma}(t) \\
& \quad \geq\left[k_{1} \xi^{\beta} q_{1}(t) z^{\beta}(t)\right]^{1 / k_{1}}\left[k_{2} \xi^{\gamma} q_{2}(t) z^{\gamma}(t)\right]^{1 / k_{2}} \\
& \quad \geq \xi^{\alpha}(t)\left[k_{1} q_{1}(t)\right]^{1 / k_{1}}\left[k_{2} q_{2}(t)\right]^{1 / k_{2}} z^{\alpha}(t) .
\end{aligned}
$$

Therefore, combining (77), (88), and (90), we obtain

$$
\begin{aligned}
& -\left(r(t)\left|z^{(n-1)}(t)\right|^{\alpha-1} z^{(n-1)}(t)\right)^{\prime} \\
& \quad \geq \overline{Q(t)} z^{\alpha}(t) \geq\left(\frac{\lambda M}{(n-2) !} t^{n-2} \delta(t)\right)^{\alpha} \overline{Q(t)} .
\end{aligned}
$$

Define the function $u$ by

$$
\begin{aligned}
u(t) & =\eta(t) r(t)\left|z^{(n-1)}(t)\right|^{\alpha-1} z^{(n-1)}(t) \\
& =-\eta(t) r(t)\left(-z^{(n-1)}(t)\right)^{\alpha}, \quad t \geq t_{1} .
\end{aligned}
$$

Then

$$
\begin{aligned}
u^{\prime}(t)= & -\eta^{\prime}(t) r(t)\left(-z^{(n-1)}(t)\right)^{\alpha} \\
& -\eta(t)\left(r(t)\left(-z^{(n-1)}(t)\right)^{\alpha}\right)^{\prime} \\
\leq & -\eta(t)\left(q_{0}(t) x^{\alpha}\left(\tau_{0}(t)\right)+q_{1}(t) x^{\beta}\left(\tau_{1}(t)\right)\right. \\
& \left.+q_{2}(t) x^{\gamma}\left(\tau_{2}(t)\right)\right) \\
\leq & -\left(\frac{\lambda M}{(n-2) !} t^{n-2} \delta(t)\right)^{\alpha} \eta(t) \overline{Q(t)} .
\end{aligned}
$$

Integrating from $t_{1}$ to $t$, we get

$$
u(t)-u\left(t_{1}\right) \leq-\int_{t_{1}}^{t}\left(\frac{\lambda M}{(n-2) !} s^{n-2} \delta(s)\right)^{\alpha} \eta(s) \overline{Q(s)} d s .
$$

Therefore,

$$
\begin{aligned}
-\eta(t) & r(t)\left(-z^{(n-1)}(t)\right)^{\alpha} \\
\leq & -\int_{t_{1}}^{t}\left(\frac{\lambda M}{(n-2) !} s^{n-2} \delta(s)\right)^{\alpha} \eta(s) \overline{Q(s)} d s
\end{aligned}
$$

that is,

$$
\begin{aligned}
& z^{(n-1)}(t) \\
& \quad \leq-\left(\frac{1}{\eta(t) r(t)} \int_{t_{1}}^{t}\left(\frac{\lambda M}{(n-2) !} s^{n-2} \delta(s)\right)^{\alpha}\right.
\end{aligned}
$$

$$
\times \eta(s) \overline{Q(s)} d s)^{1 / \alpha}
$$

Integrating the above inequality from $t_{1}$ to $l\left(l>t_{1}\right)$, we obtain

$$
\begin{gathered}
z^{(n-2)}(l)-z^{(n-2)}\left(t_{1}\right) \\
\leq-\int_{t_{1}}^{l}\left(\frac{1}{\eta(t) \mathrm{r}(t)} \int_{t_{1}}^{t}\left(\frac{\lambda M}{(n-2) !} s^{n-2} \delta(s)\right)^{\alpha}\right. \\
\times \eta(s) \overline{Q(s)} d s)^{1 / \alpha} d t .
\end{gathered}
$$

Letting $l \rightarrow \infty$ and using (73) in (97), we have $\lim _{l \rightarrow \infty} z^{(n-2)}(l)=-\infty$, which is a contradiction with the fact that $z^{(n-2)}(t)>0$. This completes the proof.

\section{Example}

In this section, we will give an example to illustrate Theorem 11.

Example 1. Consider the even order neutral delay differential equations with mixed nonlinearities:

$$
\begin{gathered}
\left(t^{\theta}\left|z^{(n-1)}(t)\right|^{1 / 2} z^{(n-1)}(t)\right)^{\prime}+\frac{1}{t}\left|x\left(\tau_{0}(t)\right)\right|^{1 / 2} x\left(\tau_{0}(t)\right) \\
+\frac{1}{t^{3 / 2}}\left|x\left(\tau_{1}(t)\right)\right|^{-(1 / 2)} x\left(\tau_{1}(t)\right) \\
+\frac{1}{t^{3 / 4}}\left|x\left(\tau_{2}(t)\right)\right| x\left(\tau_{2}(t)\right)=0 \\
t \geq t_{0},
\end{gathered}
$$

where $z(t)=x(t)+1 / 2 x(\sigma(t))$ and $n$ is even integer.

Set $r(t)=t^{\theta}, p(t)=1 / 2, q_{0}(t)=1 / t, q_{1}(t)=b / t^{3 / 2}$, $q_{2}(t)=c / t^{3 / 4}, \gamma=2, \alpha=2 / 3, \beta=1 / 2$, and $\theta \leq 2 / 3$. Then (2) holds, $k_{1}=3$, and $k_{2}=3 / 2$. 
Take $\rho(t)=1$. It is easy to show that

$$
\begin{aligned}
\limsup _{t \rightarrow \infty} \int_{t_{1}}^{t}(\rho(s) \overline{Q(s)} & r(s)\left(\rho^{\prime}(s)\right)^{\alpha+1} \\
& \left.-\frac{(\alpha+1)^{\alpha+1}\left(f(n, \lambda) \rho(s) \tau^{n-2}(s) \tau^{\prime}(s)\right)^{\alpha}}{(\alpha)}\right) d s \\
=\limsup _{t \rightarrow \infty} \int_{t_{1}}^{t}\left(\frac{1}{2}\right)^{3 / 2} & \quad \times\left\{\frac{1}{t}+\left(3 \cdot \frac{1}{t^{3 / 2}}\right)^{1 / 3}\left(\frac{3}{2} \cdot \frac{1}{t^{3 / 4}}\right)^{2 / 3}\right\} d s=\infty .
\end{aligned}
$$

Hence, by Theorem 11, every solution of (98) is oscillatory.

\section{Conflict of Interests}

The authors declare that there is no conflict of interests regarding the publication of this paper.

\section{Acknowledgments}

The authors sincerely thank the reviewers for their valuable suggestions and useful comments that have led to the present improved version of the original paper. This research is supported by the Natural Science Foundation of China (61374074), Natural Science Outstanding Youth Foundation of Shandong Province (JQ201119), and Shandong Provincial Natural Science Foundation (ZR2012AM009 and ZR2011AL007).

\section{References}

[1] R. P. Agarwal, S. R. Grace, and D. O’Regan, Oscillation Theory for Difference and Functional Differential Equations, Kluwer Academic, Dordrecht, The Netherlands, 2000.

[2] R. P. Agarwal, S. R. Grace, and D. O’Regan, "Oscillation criteria for certain $n$th order differential equations with deviating arguments," Journal of Mathematical Analysis and Applications, vol. 262, no. 2, pp. 601-622, 2001.

[3] R. P. Agarwal and S. R. Grace, "Oscillation theorems for certain functional differential equations of higher order," Mathematical and Computer Modelling, vol. 39, no. 9-10, pp. 1185-1194, 2004.

[4] Y. Sun and F. Meng, "Note on the paper of Džurina and Stavroulakis," Applied Mathematics and Computation, vol. 174, pp. 1634-1641, 2006.

[5] C. H. G. Philos, "A new criteria for the oscillatory and asymptotic behavior of delay differential equations," Bulletin of the Polish Academy of Sciences Mathematics, vol. 39, pp. 61-64, 1981.

[6] Y. G. Sun and F. W. Meng, "Oscillation of second-order delay differential equations with mixed nonlinearities," Applied Mathematics and Computation, vol. 207, no. 1, pp. 135-139, 2009.

[7] Z. Xu and Y. Xia, "Integral averaging technique and oscillation of certain even order delay differential equations," Journal of Mathematical Analysis and Applications, vol. 292, no. 1, pp. 238246, 2004.
[8] C. Zhang, T. Li, B. Sun, and E. Thandapani, "On the oscillation of higher-order half-linear delay differential equations," Applied Mathematics Letters, vol. 24, no. 9, pp. 1618-1621, 2011.

[9] Z. Han, T. Li, S. Sun, and Y. Sun, "Remarks on the paper [Appl. Math. Comput. 207 (2009) 388-396]," Applied Mathematics and Computation, vol. 215, no. 11, pp. 3998-4007, 2010.

[10] R. Xu and F. Meng, "New Kamenev-type oscillation criteria for second order neutral nonlinear differential equations," Applied Mathematics and Computation, vol. 188, no. 2, pp. 1364-1370, 2007.

[11] B. Karpuz, J. V. Manojlović, Ö. Öcalan, and Y. Shoukaku, "Oscillation criteria for a class of second-order neutral delay differential equations," Applied Mathematics and Computation, vol. 210, no. 2, pp. 303-312, 2009.

[12] L. H. Liu and Y. Z. Bai, "New oscillation criteria for secondorder nonlinear neutral delay differential equations," Journal of Computational and Applied Mathematics, vol. 231, no. 2, pp. 657-663, 2009.

[13] Z. Han, T. Li, S. Sun, and W. Chen, "Oscillation criteria for second-order nonlinear neutral delay differential equations," Advances in Difference Equations, vol. 2010, Article ID 763278, 23 pages, 2010.

[14] T. Li, Z. Han, C. Zhang, and S. Sun, "On the oscillation of second-order Emden-Fowler neutral differential equations," Journal of Applied Mathematics and Computing, vol. 37, no. 1-2, pp. 601-610, 2011.

[15] Z. Han, T. Li, C. Zhang, and Y. Sun, "Oscillation criteria for certain second-order nonlinear neutral differential equations of mixed type," Abstract and Applied Analysis, vol. 2011, Article ID 387483, 9 pages, 2011.

[16] S. Sun, T. Li, Z. Han, and Y. Sun, "Oscillation of secondorder neutral functional differential equations with mixed nonlinearities," Abstract and Applied Analysis, vol. 2011, Article ID 927690, 15 pages, 2011.

[17] Z. Han, T. Li, S. Sun, and W. Chen, "Oscillation criteria for second-order nonlinear neutral delay differential equations," Advances in Difference Equations, vol. 2010, Article ID 763278, 8 pages, 2010.

[18] T. Li, Z. Han, C. Zhang, and H. Li, "Oscillation criteria for second-order superlinear neutral differential equations," Abstract and Applied Analysis, vol. 2011, Article ID 367541, 17 pages, 2011.

[19] F. Meng and R. Xu, "Oscillation criteria for certain even order quasi-linear neutral differential equations with deviating arguments," Applied Mathematics and Computation, vol. 190, no. 1, pp. 458-464, 2007.

[20] T. Li, Z. Han, P. Zhao, and S. Sun, "Oscillation of even-order neutral delay differential equations," Advances in Difference Equations, vol. 2010, Article ID 184180, 9 pages, 2010.

[21] Y. Sun and Z. Han, "Oscillation criteria for even order halflinear neutral delay differential equations with damping," in Proceedings of the 5th International Congress on Mathematical Biology (ICMB '11), vol. 1, pp. 120-124, World Academic Press, Nanjing, China, 2011.

[22] Y. Sun, Z. Han, S. Sun, and C. Zhang, "Oscillation criteria for even order nonlinear neutral differential equations," Electronic Journal of Qualitative Theory of Differential Equations, vol. 2012, no. 30, pp. 1-12, 2012.

[23] C. Zhang, R. P. Agarwal, M. Bohner, and T. Li, "New results for oscillatory behavior of even-order half-linear delay differential equations," Applied Mathematics Letters, vol. 26, no. 2, pp. 179183, 2013. 
[24] R. P. Agarwal, M. Bohner, and T. Li, "A new approach in the study of oscillatory behavior of even-order neutral delay differential equations," Applied Mathematics and Computation, vol. 225, pp. 787-794, 2013.

[25] C. Zhang, R. P. Agarwal, and T. Li, "Oscillation and asymptotic behavior of higher-order delay differential equations with $p$ Laplacian like operators," Journal of Mathematical Analysis and Applications, vol. 409, no. 2, pp. 1093-1106, 2014. 


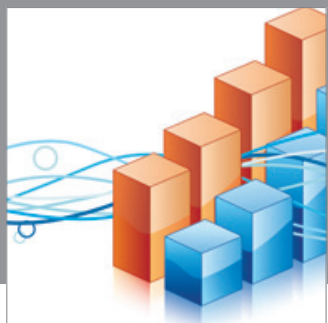

Advances in

Operations Research

mansans

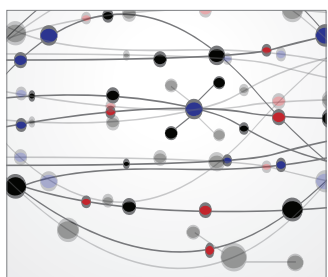

The Scientific World Journal
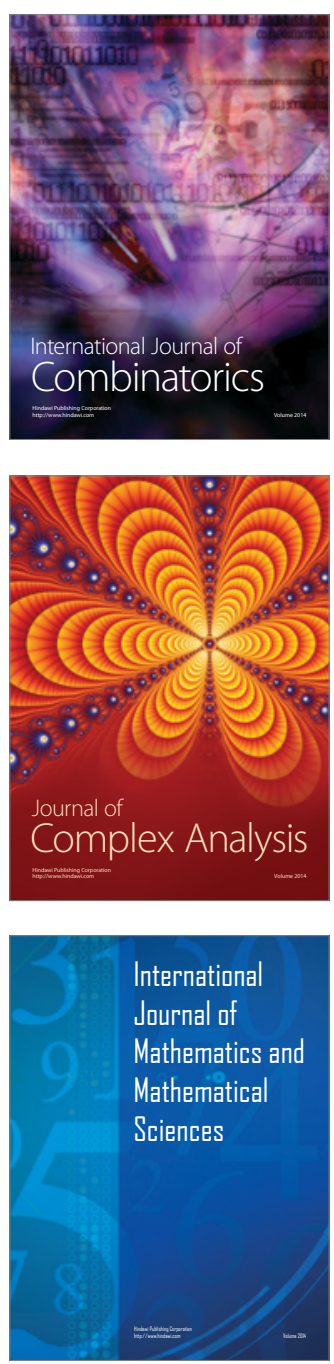
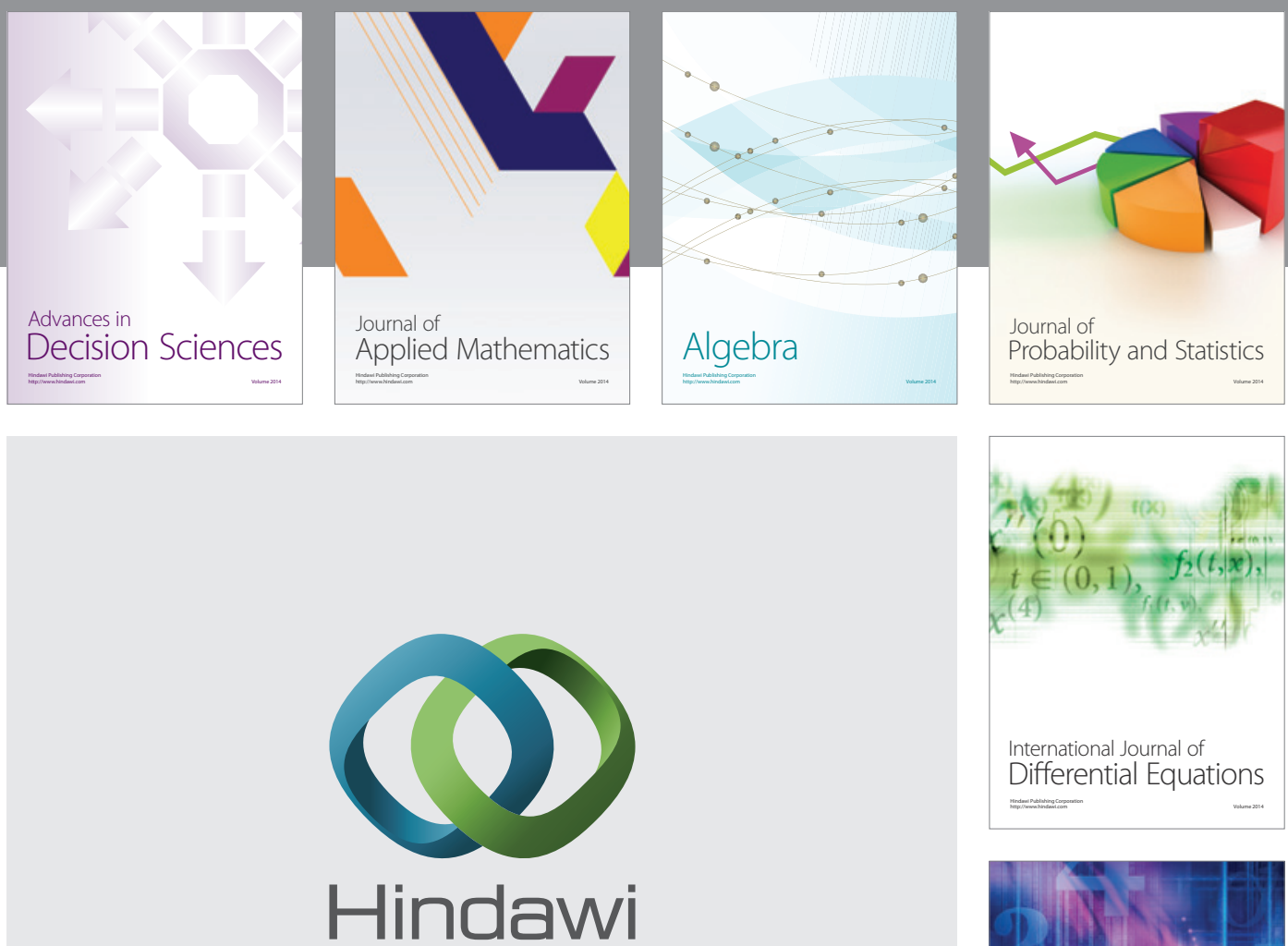

Submit your manuscripts at http://www.hindawi.com
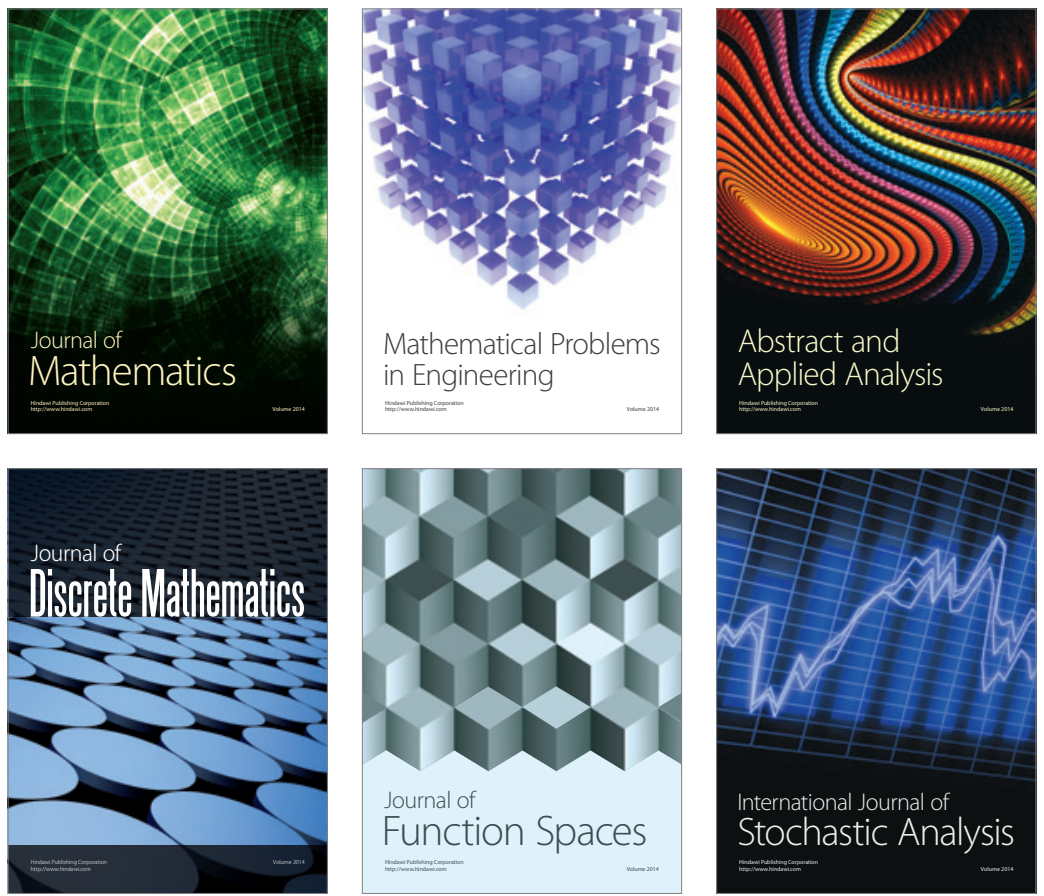

Journal of

Function Spaces

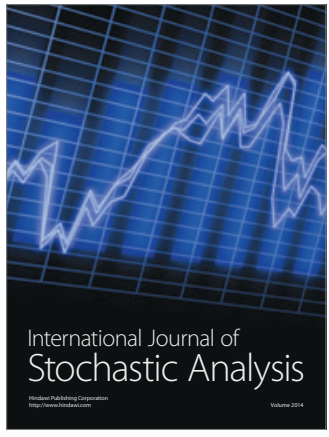

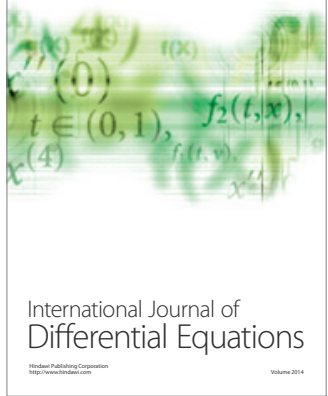
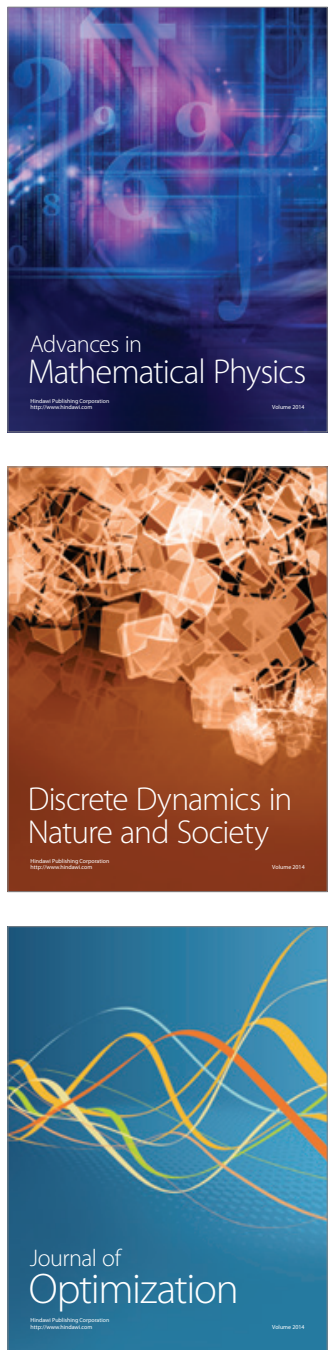